\title{
Lasergestützte Meßtechnik
}

\author{
H.J. Tiziani
}

Die meßtechnische Erfassung von Abständen und mechanischen Größen, wie Form, Verformung, Dehnung, Spannung oder Schwingungen, erfolgt heute noch vielfach mit taktilen Sensoren, die die zu messende mechanische Größe in elektrische Signale umwandeln. Die vorwiegend eingesetzten Sensoren sind kapazitive und induktive Aufnehmer und Dehnmeßstreifen. Die Grenzen dieser Verfahren werden aber schon erreicht. Zukünftig werden noch höhere Anforderungen an die Meßtechnik gestellt werden. Dabei stehen besonders berührungslose Meßverfahren im Vordergrund.

Steigerung der Leistungsfähigkeit, Lebensdauer und $\mathrm{Zu}$ verlässigkeit der Maschinen - bei reduzierter Umweltbelastung -, aber auch die Fortschritte in der Fertigungstechnik und die Einführung neuer Werkstoffe, wie Keramik und Faserverbundwerkstoffe, machen neue MeBund Prüfverfahren erforderlich.

Seit der Einführung des Lasers 1960 haben sich die optischen, insbesondere die lasertechnischen Meß- und Prüfverfahren als nützliche berührungslose, zerstörungsfreie Techniken zur Erfassung mechanischer Größen erwiesen. Sie eignen sich zur Abstandsmessung, Verformungs-, Dehnungs- und Spannungsanalyse, aber auch zur Schwingungsmessung, Oberflächen- und Formkontrolle sowie zur Mikroprofil- und integralen Rauheitsmessung. Die wichtigsten Verfahren basieren auf der Interferometrie, holografischen Interferometrie, Speckle-Interferometrie und Speckle-Fotografie sowie der Anwendung der Moiré-Technik. Die Meßdaten werden meistens in Form von Streifensystemen aufgezeichnet, die als Höhenschichtlinien der jeweiligen Meßgröße interpretiert werden können.

Die Interferometrie wurde schon lange vor der Erfindung des Lasers zur Untersuchung durchsichtiger und reflektierender Objekte eingesetzt. Der industrielle Einsatz erforderte aber eine geeignete Laserlichtquelle und die opto-elektronische Bestimmung der Wellenfronten, bzw. der Interferenzphase, auf Bruchteile der Wellenlänge des verwendeten Lichtes. Damit können auch der Bereich und die Meßgenauigkeit wesentlich gesteigert werden. Interferometrie mit Laserlicht eignet sich auch zur Untersuchung rauher Oberflächen mit Hilfe der Holografie oder durch geschickte Ausnutzung der statistischen Eigenschaften des gestreuten Lichtes, den sogenannten Speckles. Darauf basieren die in diesem Heft diskutierten Speckle-Abstands-, sowie Rauheitsmeßverfahren. Auch Streulichtmessungen ergeben nützliche Information zur Abschätzung der Oberflächenrauheit.
Die Laser-Interferometrie und Doppler-shift-Schwingungsmessung eignen sich zur punktweisen Schwingungsmessung in einem großen Frequenzbereich. In der Doppler-Verschiebungsmeßtechnik und der Heterodyn-LaserInterferometrie wird, mit unterschiedlichen Methoden, im Referenz- oder Prüfstrahl die Frequenz um mehr als die zu erwartende Frequenzänderung des Meßsignals verschoben $(100 \mathrm{kHz}$ bis $40 \mathrm{MHz})$. Für gezielt eingeführte Frequenzschiebung eignen sich akusto-optische Modulatoren oder das magnetische Feld im Laser-Resonator (Zeeman-Aufteilung) oder rotierende Gitter, aber auch polarisationsoptische Verfahren. Die durch das mechanisch schwingende Objekt bewirkte Phasenmodulation kann durch geeignete elektronische Phasen- oder Frequenzmodulatoren in ein zur Schwingungsamplitude (optische Phase) oder Schwingungsgeschwindigkeit (Doppler-Verschiebung) proportionales Signal umgewandelt werden. Dadurch sind sehr genaue quantitative Schwingungsmessungen, tangential oder senkrecht zur Oberfläche, möglich. Das Heterodyn-Verfahren eignet sich auch zur genauen Abstands- und Mikroprofilmessung.

Die holografische Interferometrie und die Speckle-Interferometrie basieren auf der Messung der Wegänderung und liefern eine bildhafte Darstellung der Verformung bzw. der Schwingungsamplituden von Werkstücken.

Obwohl die Euphorie der ersten Jahre bei der Holografie einer gewissen Ernüchterung Platz machte, sind inzwischen interessante Einsatzmöglichkeiten in der Industrie gefunden worden, z.B. zur Mithilfe bei der Entwicklung von Maschinenteilen, Turbinen, Geräusch- und Schwingungsuntersuchung, zur Serienprüfung von Flugzeugreifen. Die Meßempfindlichkeit der Verfahren ist abhängig von der Wellenlänge des verwendeten Lichtes. Der Unterschied zwischen der holografischen und der Speckle-Interferometrie besteht im wesentlichen darin, daß die Auflösung des Speichers für die Aufzeichnung bei der Holografie wesentlich höher sein $\mathrm{muß}(\geqq 400 \mathrm{lp} / \mathrm{mm}$ ) als bei der Specklefeld-Registrierung, wo TV-Systeme ausreichen. Diese zweidimensionalen Bilder werden vorzugsweise mit Hilfe der automatischen Streifenauswertung analysiert. Festkörperdetektoren, aber auch die Entwicklung der Mikroprozessoren haben zur kostengünstigen Auswertung von Interferenzstreifen geführt.

Die quantitativen Quasi-Heterodyn-Auswerteverfahren mit Bildverarbeitung bzw. echter Heterodynphasenmessung erlauben bei gutem Streifenkontrast Auswertungen von $1 / 100$ bzw. $1 / 1000$ Streifenabstand. 
Die Verfahren eignen sich auch zur Höhenschichtliniendarstellung von kontinuierlich variierenden 3D-Objekten.

Die Speckle-Fotografie bedient sich der Speckleverschiebung, bedingt durch die Objektverschiebung oder Verkippung. Dabei ist die Meßempfindlichkeit in der Gegend der Auflösung der fotografischen Abbildung. Rechnergestützte Auswertungen ergeben für tangentiale Oberflächenverschiebungen eine relative Genauigkeit von $1 \%$.

Die Anwendung der Laser-Meßtechnik, besonders durch computergestützte quantitative Auswertung, hat folgende Vorteile:

- bessere konstruktive Auslegung von Maschinen, Fahrzeugen, Turbinen etc., geringerer Verschleiß, höhere Lebensdauer;

- durch Einsparung von schwingungsdämpfenden Materialien geringeres Gewicht, Rohstoffersparnis und geringerer Energieverbrauch;

- Reduzierung des Lärmpegels;

- größere Betriebssicherheit an beanspruchten Teilen, weniger Materialaufwand und Ersparnis von Rohstoffen;

- durch optimierte Auslegung von Bauteilen Wettbewerbsvorteile.

Die optische Korrelation kann zum Formvergleich und zur Vollständigkeitsprüfung benutzt werden. Allerdings begrenzt das bei kohärenter Beleuchtung auftretende Specklemuster die industrielle Anwendung auf Spezialfälle. Geeignete Wandler haben aber in jüngster Zeit der analog-optischen Korrelation neuen Aufwind gegeben. Hybride Systeme und eine geeignete Kombination der analogen und digitalen intelligenten Auswerteverfahren können für die Zukunft von Bedeutung sein.

Laseroptische Sensoren werden zukünftig häufiger im Automatisierungsproze $B$ eingesetzt werden zur Vollständigkeits- und Abstandsprüfung. Aber auch der Roboter wird sich zukünftig vermehrt der Lasermeßtechnik bedienen. Auf Lichtschnitt, Triangulation, Laufzeitmessung und Korrelation basierende Methoden werden weiter entwikkelt. Hier bahnen sich neue Verfahren der LasermeBtechnik an. Automatisierte Meßsysteme haben noch ein weites Anwendungsfeld. Dazu wird auch der Einsatz neuer Halbleiter-Laser beitragen.

Mit der Entwicklung des Lasers ist auch die optische Spektroskopie in bezug auf Auflösung und Empfindlichkeit wesentlich gesteigert worden. Ein breites Einsatzgebiet der laserspektroskopischen Verfahren wurde bei der Gasanalyse erschlossen. Anwendungen im Bereich des Umweltschutzes, zur Untersuchung und Optimierung von Verbrennungsvorgängen in Motoren und technischen Flammen, zur Überwachung und Steuerung von Prozeßabläufen sind nur einige Möglichkeiten. Darauf soll aber in diesem Heft nicht eingegangen werden, das nur einen Ausschnitt der verschiedenen Möglichkeiten und Aktivitäten wiedergeben kann. 